\title{
Cost of Relapse Management in Patients with Schizophrenia in Italy and Spain: Comparison Between Lurasidone and Quetiapine XR
}

\author{
Umberto Restelli ${ }^{1,2}\left(\right.$ D $\cdot$ Manuel García-Goñi ${ }^{3} \cdot$ Michal Lew-Starowicz ${ }^{4,5} \cdot$ Pawel Mierzejewski $^{6} \cdot$ Sofia Silvola $^{1}$. \\ Jacqueline Mayoral-van Son ${ }^{7} \cdot$ Davide Croce $^{1} \cdot$ Paola Rocca $^{8} \cdot$ Benedicto Crespo-Facorro $^{7}$
}

Published online: 9 July 2020

(c) The Author(s) 2020

\begin{abstract}
Background and Objective Schizophrenia is a low-prevalence mental disorder with a global age-standardized prevalence of 21 million people (2016). Second-generation antipsychotics (lurasidone and quetiapine XR) are recommended as the firstline treatment for schizophrenia. It is interesting to investigate how the results of clinical studies translate into direct medical costs. The objective of this analysis was to assess the direct medical costs related to pharmaceutical treatments and the management of relapses in patients affected with schizophrenia treated with lurasidone (74 mg) vs quetiapine XR (300 mg) assuming the Italian and Spanish National Health Service perspective.

Methods A health economic model was developed based on a previously published model. The analysis considered direct medical costs related to the pharmacological therapies and inpatient or outpatient management of relapses (direct medical costs referred to 2019). The probability of relapses and related costs were derived from two systematic reviews. A deterministic sensitivity analysis was implemented to test the robustness of the results.

Results The use of lurasidone $(74 \mathrm{mg})$ compared with quetiapine XR $(300 \mathrm{mg})$ would lead to a reduction in direct medical costs in Italy and Spain, with a lower cost per patient of $-163.7 €(-9.0 \%)$ and $-327.2 €(-22.7 \%)$, respectively. In detail, it would lead to an increase in the cost of therapy of $+53.8 \%$ and of $+30.5 \%$ in Italy and Spain, respectively, to a decrease in the cost of relapses with hospitalization of $-135.7 \%$, and to an increase in the cost of relapses without hospitalization of $+24.5 \%$.

Conclusions The use of lurasidone (74 mg) for the treatment of patients affected with schizophrenia, compared with quetiapine XR (300 mg), would be a cost-saving strategy in the two contexts investigated assuming the National Health Service point of view.
\end{abstract}

\section{Background}

Schizophrenia is a low-prevalence mental disorder, having a global age-standardized prevalence of $0.28 \%$ (95\% uncertainty interval 0.24-0.31) in 2016, which consists of 21 million people globally affected [1]. In Europe in 2011, the median prevalence of the pathology, which tends to become chronic, was $1.2 \%$, consisting of 5 million persons [2]. The prevalence of this mental disorder is different among age

Electronic supplementary material The online version of this article (https://doi.org/10.1007/s40261-020-00944-0) contains supplementary material, which is available to authorized users.

Umberto Restelli

urestelli@gmail.com

Extended author information available on the last page of the article groups. This leads to a significant economic impact in terms of a loss of productivity of individuals and family members, as the group most affected by schizophrenia is the working age population.

Schizophrenia can be treated with conventional antipsychotics, introduced from the 1950s onwards, and atypical antipsychotics, from the 1990s onwards, with a higher demonstrated efficacy compared with conventional antipsychotics $[3,4]$.

Schizophrenia has a significant impact in terms of direct and indirect costs, for patients, caregivers, and society [5]. In USA in 2011, compared with other mental disorders, schizophrenia registered the second-longest average length of stay and the highest aggregate cost of hospitalization, which are attributable for $52 \%$ to indirect costs, $36 \%$ to direct healthcare costs, and $12 \%$ to direct non-healthcare costs [5]. In Europe, from a review published in 2018, the direct cost 


\section{Key Points}

The use of lurasidone (74 mg) compared with quetiapine XR $(300 \mathrm{mg})$ for the treatment of patients affected with schizophrenia would lead to a reduction in the direct medical costs related to the management of patients for the Italian and Spanish National Health Service, mainly owing to a reduction in relapses requiring hospitalization.

The reduction in relapses requiring inpatient management would lead to positive organizational consequences for hospitals.

per patient is between a maximum of $13,704 €$ in the Netherlands and a minimum of $533 €$ in Ukraine, with a major contribution attributable to inpatient costs and $25 \%$ of costs attributable to drugs [2].

Furthermore, in a recent Spanish study, analyzing the direct and indirect costs in a first episode of psychoses intervention program, the main findings were that the total costs during the first year of treatment after a first break of psychosis, on average per patient, were $48,354 €$, with direct healthcare costs of $13,729 €$, direct non-medical costs of $109 €$, and indirect costs of $34,515 €$, the latter representing $71.39 \%$ of the total cost [6].

A few studies in the literature have provided a cost-effectiveness analysis for patients with schizophrenia in Spain, one of the countries of interest in this investigation. For instance, Olivares et al. [7] examined the impact of switching a previous antipsychotic to long-acting injectable risperidone and Gutiérrez-Recacha et al. [8] focused on seven different types of clinical interventions at the level of the Spanish population. However, recently, to the best of our knowledge, neither Spain nor Italy has addressed the impact of one of the key and most significant drivers of direct medical and indirect costs in patients affected with schizophrenia-relapses. The choice of treatment for patients with schizophrenia may affect the likelihood of relapse. Different atypical antipsychotics can prevent or delay relapses in patients with schizophrenia [9] improving clinical outcomes, and leading to economic savings and to a more efficient allocation of resources from the societal perspective.

Antipsychotics, in particular second-generation antipsychotics, are recommended as the first-line treatment for schizophrenia [10]. Antipsychotics have been found to be a heterogeneous class of drugs with varied efficacy and tolerability profiles. Lurasidone and quetiapine XR, both are classified as second-generation antipsychotics. These two drugs have similar efficacy, but different pharmacological profiles. When comparing patients with schizophrenia treated with lurasidone and quetiapine $\mathrm{XR}$, it has been shown that those treated for 12 months with lurasidone had a statistically significantly reduced risk of hospitalization [11]. It is interesting to investigate how the results of clinical studies translate into direct medical costs.

Lurasidone is a second-generation antipsychotic that received a marketing authorization valid throughout the European Union by the European Medicines Agency on the 20 March, 2014, and is indicated for the treatment of schizophrenia in adults aged 18 years and over [12]. Lurasidone is a dopamine $\mathrm{D}_{2}$ and serotonin $5-\mathrm{HT}_{2 \mathrm{~A}}$ receptor antagonist, in addition, it has distinctive $5-\mathrm{HT}_{7}$ antagonistic activity and partial agonism at 5-HT $\mathrm{HA}_{1 \mathrm{~A}}$ [13].

Quetiapine XR received a market authorization valid throughout the European Union on the 26 August, 2010 and among its treatment indications there is "treatment of schizophrenia" [14]. It has a moderate affinity for $5-\mathrm{HT}_{2 \mathrm{~A}}$, $\alpha 1, \mathrm{M} 1$, and $\mathrm{H} 1$ receptors, and only a minor affinity for $\mathrm{D}_{2}$ and $5-\mathrm{HT}_{1 \mathrm{~A}}$ receptors [15].

The objective of our analysis is to fill the gap in the literature related to the absence of direct comparisons of these two antipsychotics from an economic perspective at a European level by assessing the direct medical costs of relapse management in patients affected with schizophrenia, and accounting for the different likelihood of experiencing relapses under different treatments. The goal is to determine whether there is evidence of economic savings for the National Health Service of Italy or Spain (both southern European universal systems) from the use of lurasidone (74 mg) compared to quetiapine XR (300 mg), which might result in health policy implications.

\section{Methods}

\subsection{Model Design}

A health economic model was developed using Microsoft Excel 2010 with the purpose of estimating the economic impact of relapses assuming the perspective of the National Health Service of Italy and Spain for patients with schizophrenia treated with lurasidone vs quetiapine XR. Starting from the model schematization described by Rajagopalan et al. [5], a deterministic decision tree model was implemented with a 1-year time horizon. A schematization of the model is reported in Fig. 1. The inputs considered in the model are the target population (eligible for treatment), clinical and economic data concerning relapses (i.e., hospitalizations and outpatient treatment), defined daily doses, and the cost of each treatment (cost of drugs). 


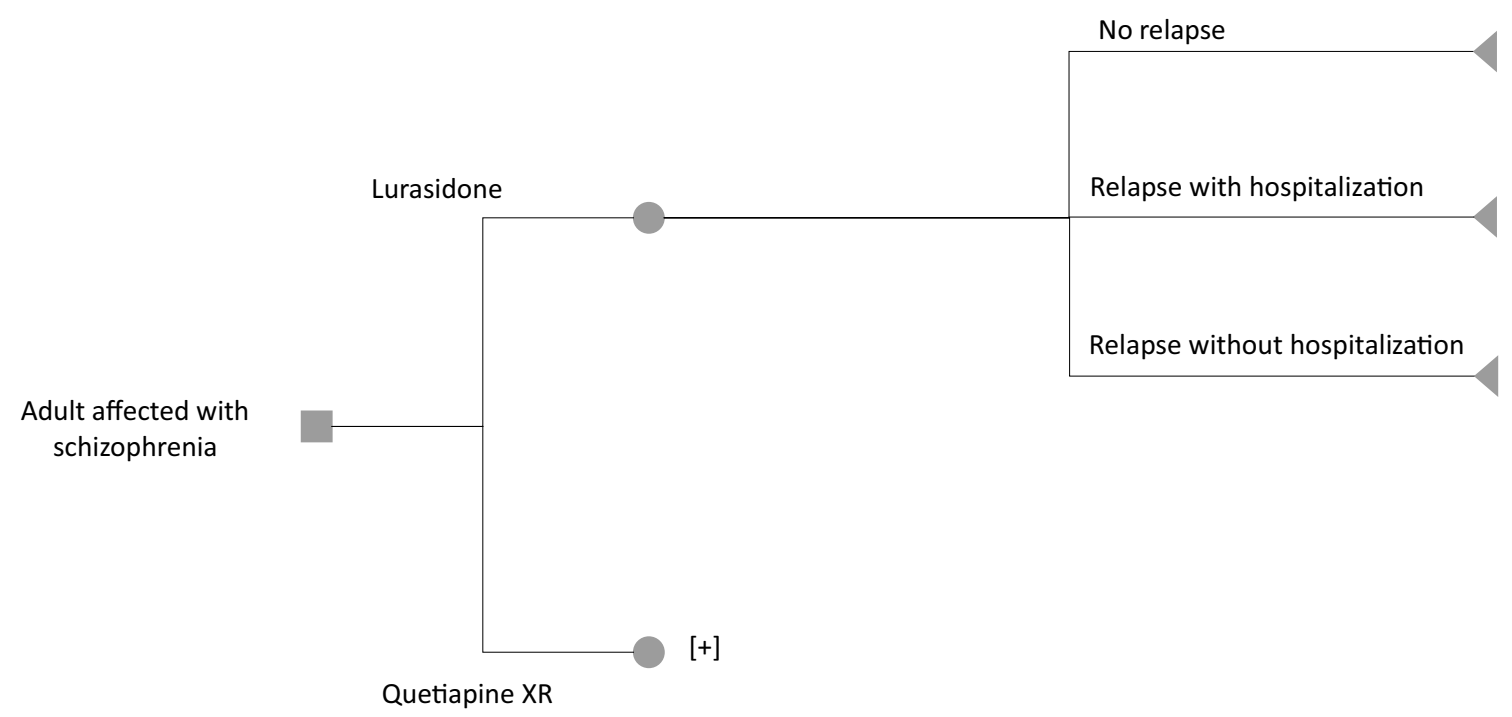

Fig. 1 Schematization of the model

\subsection{Target Population}

The target population of the analysis consists of adult individuals affected with schizophrenia treated with antipsychotics. The population considered does not reflect the real number of patients who will be treated with lurasidone or quetiapine XR in each national context, but the result will approximate the potential impact of the use of lurasidone in the case where all eligible patients are treated with lurasidone, compared with quetiapine XR. To estimate this population, the overall number of patients affected with schizophrenia in each context was considered. This is owing to the fact that a treated pediatric population is negligible in terms of numbers of patients, and that it would not be possible to estimate the number of patients not eligible to use the drugs for metabolic or other reasons because of a lack of available literature data. Furthermore, because of the stability of the prevalence of schizophrenia over the years, data referred to years before 2019 were considered as representative of 2019 .

In Italy, the target population was estimated considering the prevalence of treated patients affected with schizophrenia and other functional psychoses [16], being 35.8 per 10,000 inhabitants in 2017. Based on the Italian resident population $(60,359,546)$ on 1 January, 2019, as reported by Eurostat (2019) [17], the target population was estimated in 216,087 patients.

In Spain, the number of patients affected by schizophrenia is estimated to be equal to 400,000 [18]. Lacro and colleagues report that the mean non-adherence rate in patients with schizophrenia is $49.5 \%$ [19]. Considering that $50.5 \%$ of population is adherent to pharmacological therapies, the estimated number of patients considered in the analysis for the Spanish context is 202,000 .

\subsection{Probability of Relapse}

To estimate the probability of relapse in individuals with schizophrenia receiving treatment with lurasidone or quetiapine XR, a systematic literature review was conducted through PubMed. In accordance with PRISMA (Preferred Reporting Items for Systematic Reviews and Meta-Analyses) guidelines, the process was divided into four stages: identification, screening, eligibility, and inclusion [20]. Concerning the identification stage, the keywords used for this search were: ("lurasidone" OR "quetiapine XR") "schizophrenia". The resulting search string was: (("lurasidone hydrochloride"[MeSH Terms] OR ("lurasidone"[All Fields] AND "hydrochloride"[All Fields]) OR "lurasidone hydrochloride"[All Fields] OR "lurasidone"[All Fields]) OR (("quetiapine fumarate"[MeSH Terms] OR ("quetiapine"[All Fields] AND "fumarate"[All Fields]) OR "quetiapine fumarate"[All Fields] OR "quetiapine"[All Fields]) AND XR[All Fields])) AND ("schizophrenia"[MeSH Terms] OR "schizophrenia"[All Fields]).

To assess the probability of relapse, the eligibility criteria to identify the articles were: reference to the pathology of interest (schizophrenia), include at least one of the contexts of interest (Italy or Spain), and include a direct comparison of the two drugs included in the analysis (quetiapine XR or lurasidone). The search identified 240 documents. In accordance with the screening stage, 223 documents were excluded, not being pertinent to the subject or because written in languages other than English, Italian, or Spanish. 
Using a single database, it was not necessary to remove duplicates.

For the eligibility stage, two independent researchers reviewed the results, after the reading of the abstracts, six articles were excluded because they did not compare the two selected drugs. Of the 11 remaining articles, after reading the entire manuscripts, six of them were excluded because they did not consider relapses as an outcome measure, three because they did not compare directly the two drugs, and one was excluded because the results presented were already included in a more recent publication. Concerning the inclusion stage, only one article was considered as pertinent with the review. A schematization of the research performed is reported in Fig. 2.
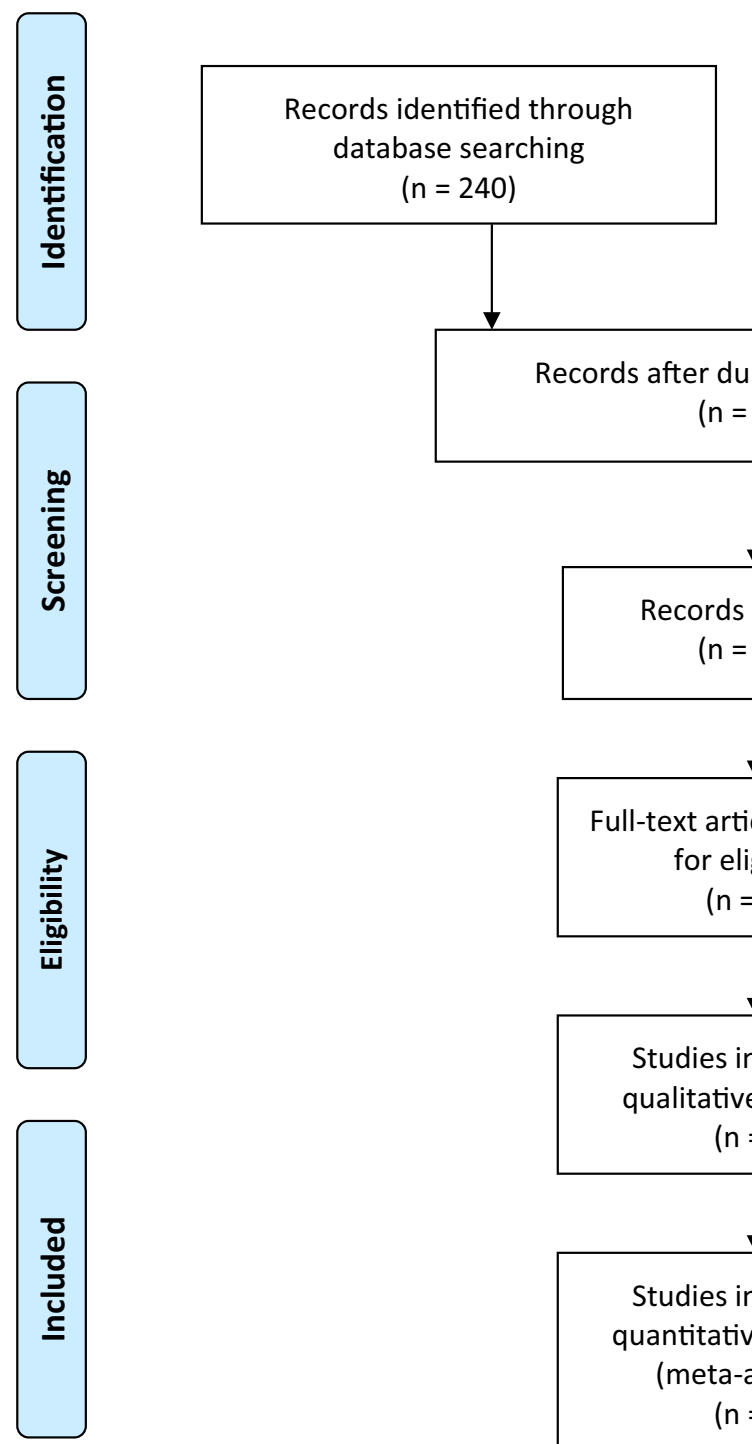

The selected study, a 12-month double-blind non-inferiority study, published by Loebel et al. [11], assessed the non-inferiority of flexibly-dosed lurasidone (37-148 mg/ day) compared with quetiapine XR (200-800 mg/day) in terms of relapse prevention, in a 12 -month period, in adult patients with chronic schizophrenia who completed a previous 6-week placebo-controlled trial. Relapse was defined as the occurrence of one criterion between: worsening of $30 \%$ or more in the Positive and Negative Syndrome Scale total score from day 42 from short-term treatment and Clinical Global Impression Scale-Severity of Illness score $\geq 3$, re-hospitalization or aggravation of psychosis, emergency related to suicidal or homicidal ideation, or risk of harm. Results showed that lurasidone had a $23.7 \%$ probability

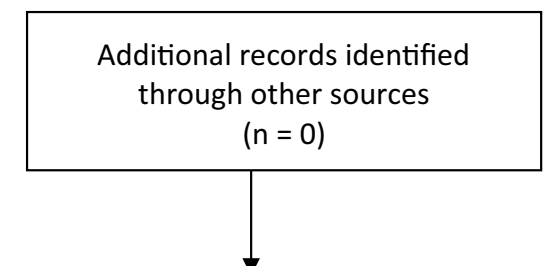

Records after duplicates removed

$(n=240)$
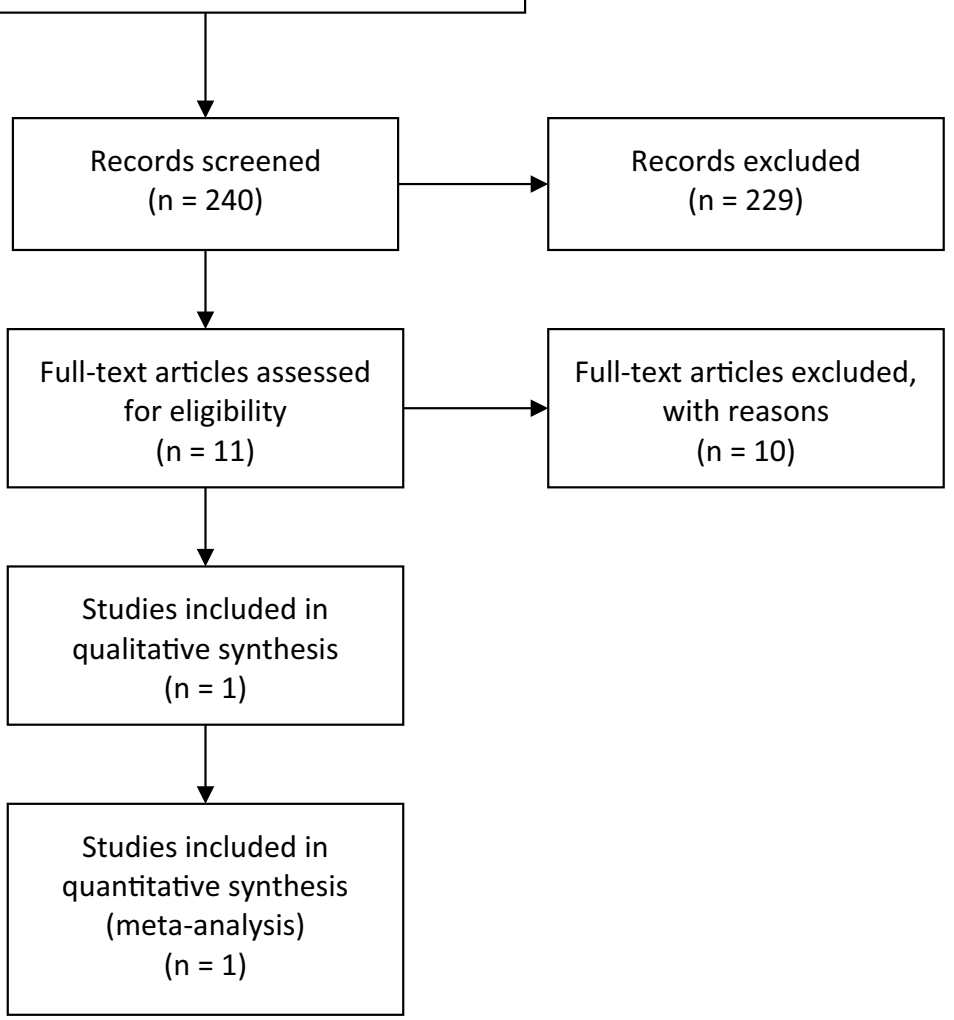

Fig. 2 PRISMA flow diagram of literature review to estimate the probability of relapse in individuals with schizophrenia receiving treatment with lurasidone or quetiapine XR 
of relapse and quetiapine XR had a $33.6 \%$ probability of relapse, at 12 months, with a $27.2 \%$ reduction in relapse risk for lurasidone, which demonstrated non-inferiority in time to relapse and probability of relapse. Furthermore, lurasidone showed a significantly lower probability of hospitalization at 12 months compared with quetiapine XR $(9.8 \%$ vs $23.1 \%$; $p<0.05$ ).

The probability of relapses managed in inpatients at 12 months was considered as the probability of hospitalization at 12 months, while the probability of relapses managed in outpatients at 12 months was derived as the difference of the probability of relapse at 12 months and of the probability of hospitalization at 12 months. Data related to relapses probability are reported in Table 1.

\subsection{Direct Medical Costs}

The costs considered in the analysis are direct medical costs related to the cost of antipsychotics and to the cost of relapses. To assess the direct medical costs related to relapses, a systematic literature review was conducted in accordance with PRISMA guidelines [20]. The identification stage was conducted using the following keywords on the search engine PubMed: "cost" and "schizophrenia" and the resulting search string was: (("economics"[Subheading] OR “economics”[All Fields] OR "cost”[All Fields] OR "costs and cost analysis"[MeSH Terms] OR ("costs"[All Fields] AND "cost"[All Fields] AND "analysis"[All Fields]) OR "costs and cost analysis"[All Fields]) AND ("schizophrenia"[MeSH Terms] OR "schizophrenia"[All Fields])).

To assess direct medical cost related to relapses the eligibility criteria to identify the articles were: reference to the pathology of interest (schizophrenia), include at least one of the contexts of interest (Italy or Spain) and include quantitative data of direct health costs related to relapses. Two hundred and fifty-six articles were found, of which 222 articles were excluded from reading the title during the screening stage, not being pertinent to the topic, or not being written in any of the three languages considered (as explained above). The eligibility stage was conducted starting from the reading of the abstract, after which 19 articles were excluded as they did not report economic analysis conducted in either of the countries considered: Italy and Spain. Of the remaining 15 articles, after a thorough reading, four relevant documents were considered, excluding 11 articles because the costs related to the disease were not assessed, neither were the relapses considered in the analysis. Looking carefully into the details, two articles reported an average cost at a European level, six of them were not focused on the topic of interest, and three studies did not consider relapses.

Moreover, after an analysis of the four relevant studies identified, three documents were excluded because of the lack of an explicit economic evaluation of relapses. In detail, the overall costs related to patient management were reported, not providing details on the cost of relapses. During the inclusion stage, one article was selected as pertinent to the review. A schematization of the literature review is reported in Fig. 3.

The article selected is the analysis conducted by Dilla and colleagues that compared the costs associated with the two therapies, among which neither lurasidone nor quetiapine XR was considered [21]. The cost of relapse has been obtained from a cost-effectiveness study that estimated the value over a 5-year period adopting the point of view of the Spanish National Health Service. The study reports inpatient and outpatient costs of relapse management referred to year 2011 (original data referred to year 2000). Data were obtained from a retrospective review with 200 records of patients from eight Spanish hospitals that considered fixed costs (medical, nursing, and other staff, room and catering, allocated common services costs, and other services), diagnostic tests, and drug treatment [22]. The results showed costs associated with relapse management for the Spanish Healthcare Service of $4226.91 €$ for inpatient activities and $884.38 €$ for outpatient activities, assuming hospitalization as a proxy for relapse.

Because of the lack of results concerning the issue of the economic impact of relapses in Italy, a further literature review was performed. The research focused on peerreviewed health economics journals related to the Italian context, in details: Clinico Economics-Italian Articles on Outcomes Research, Italian Journal of Public Health, Global \& Regional Health Technology Assessment-Italian, Farmeconomia-Health Economics and Therapeutic

Table 1 Clinical data: hospitalization and relapses

\begin{tabular}{lccc}
\hline & Lurasidone $(\%)$ & Quetiapine XR (\%) & Reference \\
\hline Probability of relapse at 12 months & 23.70 & 33.60 & Loebel et al. [11] \\
Probability of hospitalization at 12 months & 9.80 & 23.10 & Loebel et al. [11] \\
$\begin{array}{l}\text { Probability of relapse managed in an outpatient setting at } \\
\text { 12 months }\end{array}$ & $13.90^{\mathrm{a}}$ & $10.50^{\mathrm{a}}$ & \\
\hline
\end{tabular}

${ }^{a}$ Calculated as the difference of the probability of relapse at 12 months and of the probability of hospitalization at 12 months 


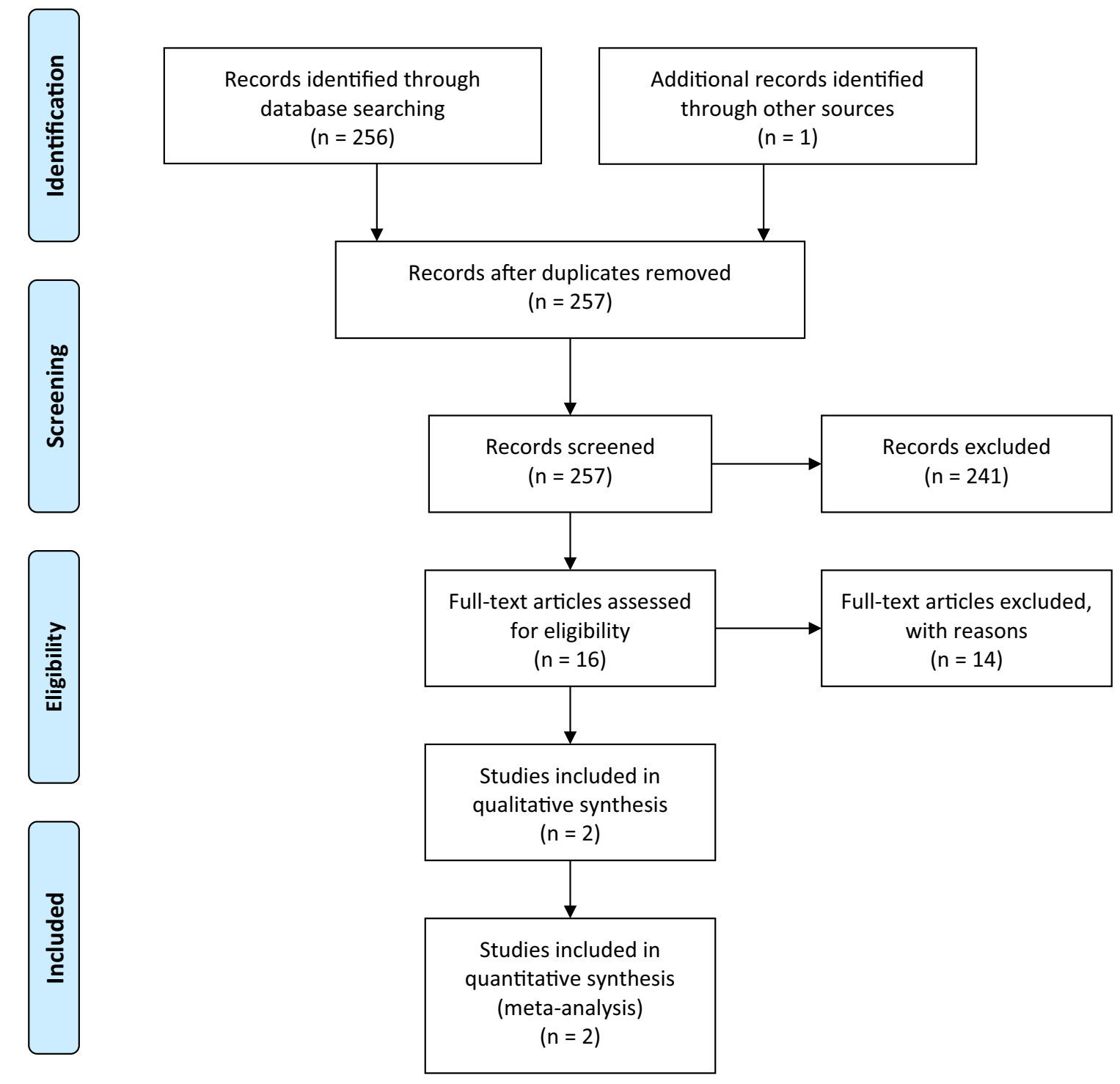

Fig. 3 PRISMA flow diagram of literature review to assess the direct medical costs related to relapses in individuals with schizophrenia

Pathways. The research identified an additional article reporting costs associated with schizophrenia, in particular related to the event of relapse, in the Italian context.

Berto et al. evaluated with a cost-effectiveness analysis the effects of schizophrenia in monetary terms from the perspective of the Italian National Health Service in a 52-week time horizon [23]. The analysis reports the cost of relapses with and without hospitalization related to five different drugs; however, the document does not report details on the calculation method. The mean weighted cost of relapses with hospitalization is 4749.25 $€$ and $1908.04 €$ without hospitalization.

The cost of relapses in each arm was calculated using the probabilities of relapse at 12 months and the costs of the management in the two countries for which they are available, inflated at year 2019 using an inflation percentage change relative to average consumer prices [24]. It is important to underline the fact that the cost of relapse management in Italy and Spain might have changed since the date of publication of the analyses published in the literature (before 2010). However, the uncertainty over this aspect has been managed in the sensitivity analysis.

The annual cost of lurasidone and quetiapine XR considered is the price to the public. The posology considered in the analysis is $74 \mathrm{mg}$ for lurasidone and the equivalent dose of quetiapine XR of $300 \mathrm{mg}$, as reported by Leucht and colleagues [25]. Costs considered in the analysis are summarized in Table 2 (i.e., cost per relapse, and cost per tablet and mean annual cost per patient). 
Table 2 Costs considered in the analysis (2019 value)

\begin{tabular}{lrr}
\hline & Italy $(€)$ & \multicolumn{1}{c}{$\begin{array}{l}\text { Spain } \\
(€)\end{array}$} \\
\hline $\begin{array}{l}\text { Cost of relapse management with } \\
\text { hospitalization }\end{array}$ & 5628.2 & 4657.7 \\
$\begin{array}{l}\text { Cost of relapse management without } \\
\text { hospitalization }\end{array}$ & 2239.9 & 974.5 \\
Lurasidone & & \\
Price per tablet (74 mg) & 2.59 & 2.33 \\
Annual cost per patient & 946.00 & 850.58 \\
Quetiapine XR & & \\
Price per tablet (300 mg) & 1.20 & 1.62 \\
Annual cost per patient & 437.27 & 591.42 \\
\hline
\end{tabular}

\subsection{Sensitivity Analysis}

A sensitivity analysis was conducted varying the following parameters: probability of relapses managed with a hospitalization $-20 \%$ (to represent the different clinical approach in the management of relapses in previous years compared with the year of the publication of Loebel and colleagues), $\pm 20 \%$ of the cost of relapses, $\pm 10 \%$ of the cost of lurasidone, and $-40 \%$ of the target population.

\section{Results}

The results of the analysis, in terms of cost per therapy, relapse with hospitalization, and relapse without hospitalization are presented in Table 3. The use of lurasidone ( $74 \mathrm{mg}$ ) compared with quetiapine XR (300 mg) would lead to a reduction in direct medical costs in Italy and Spain, with a lower cost per patient of $-163.7 €$ and $-327.2 €$, respectively. The highest impact on total cost is related to the cost of the therapy in both contexts. Concerning the Italian context, the cost of therapy with lurasidone (74 mg) compared to quetiapine XR (300 mg) registered an increase of 109,930,325 $€(+53.8 \%)$, the cost of relapses with hospitalization a decrease of $-161,741,917$ $(-135.7 \%)$, and the cost of relapses without hospitalization an increase of $16,456,757 €(+24.5 \%)$ with a total cost reduction of $-35,364,834(-9.0 \%)$. In the Spanish context, the use of lurasidone (74 mg) compared with quetiapine XR (300 mg) is associated with an increase of $52,350,055 €(+30.5 \%)$ of the cost of therapy, with a decrease of $-125,132,927 €$ $(-135.7 \%)$ of the cost of relapses with hospitalization, and with an increase of $6,692,908 €(+24.5 \%)$ of the cost of relapses without hospitalization, with a total cost reduction of $-66,089,974(-22.7 \%)$.

The results of the sensitivity analysis (reported in Table 4 and in Figs. 4, 5) show a lower and maximum total cost difference of $-1.8 \%$ and $-15.0 \%$ in Italy and of $-15.9 \%$ and $-28.5 \%$ in Spain. In both contexts, the scenario that leads to a lower cost difference is the scenario that considers a cost per-relapse reduction of $-20 \%$, while the scenario that leads to the wider cost difference is the scenario that considers an increase of the cost of relapses of $+20 \%$.

\section{Discussion}

The results of the analysis presented show a reduction in annual per capita direct medical costs related to the use of lurasidone (74 mg) compared with quetiapine XR (300 mg) both in Italy and Spain equal to $-163.7 €$ and $-327.2 €$, respectively. The sensitivity analysis confirmed the robustness of the results.

The model used in the analysis is an adaptation of that implemented by Rajagopalan and colleagues, who investigated the financial impact on schizophrenia-related relapses of the use of lurasidone vs quetiapine XR in the US context [5]. The analysis conducted in this context showed a wider difference related to the higher cost per hospitalization compared with the present analysis. Lurasidone would have led

Table 3 Results of the analysis

\begin{tabular}{lllll}
\hline Context & Cost category & Lurasidone $(€)$ & Quetiapine XR $(€)$ & $\Delta(€)$ \\
\hline Italy & Therapy & $204,418,688$ & $94,488,362$ & $+109,930,325$ \\
& Relapse with hospitalization & $119,185,623$ & $280,937,539$ & $-161,751,917$ \\
& Relapse without hospitalization & $67,279,095$ & $50,822,338$ & $+16,456,757$ \\
& Total cost & $390,883,406$ & $426,248,240$ & $-35,364,834$ \\
& Cost per patient & 1808.9 & 1972.6 & -163.7 \\
Spain & Therapy & $171,817,232$ & $119,467,177$ & $+52,350,055$ \\
& Relapse with hospitalization & $92,203,217$ & $217,336,154$ & $-125,132,937$ \\
& Relapse without hospitalization & $27,362,181$ & $20,669,273$ & $+6,692,908$ \\
& Total cost & $291,382,630$ & $357,472,603$ & $-66,089,974$ \\
& Cost per patient & 1442.5 & 1769.7 & -327.2 \\
\hline
\end{tabular}


Table 4 Results of the sensitivity analysis

\begin{tabular}{|c|c|c|c|c|c|}
\hline Context & Scenario & Cost & Lurasidone $(€)$ & Quetiapine XR $(€)$ & $\Delta(€)$ \\
\hline \multirow[t]{12}{*}{ Italy } & \multirow{2}{*}{$\begin{array}{l}\text { Probability of relapses managed } \\
\text { with a hospitalization }-20 \%\end{array}$} & Total cost & $376,533,118$ & $392,422,561$ & $-15,889,443$ \\
\hline & & Cost per patient & 1742.5 & 1816.0 & -73.5 \\
\hline & \multirow[t]{2}{*}{ Cost of relapses $+20 \%$} & Total cost & $428,176,349$ & $492,600,215$ & $-64,423,866$ \\
\hline & & Cost per patient & 1981.5 & 2279.6 & -298.1 \\
\hline & \multirow[t]{2}{*}{ Cost of relapses $-20 \%$} & Total cost & $353,590,462$ & $359,896,264$ & $-6305,802$ \\
\hline & & Cost per patient & 1636.3 & 1665.5 & -29.2 \\
\hline & \multirow[t]{2}{*}{ Cost of lurasidone $+10 \%$} & Total cost & $411,325,274$ & $426,248,240$ & $-14,922,965$ \\
\hline & & Cost per patient & 1903.5 & 1972.6 & -69.1 \\
\hline & \multirow[t]{2}{*}{ Cost of lurasidone $-10 \%$} & Total cost & $370,441,537$ & $426,248,240$ & $-55,806,703$ \\
\hline & & Cost per patient & 1714.3 & 1972.6 & -258.3 \\
\hline & \multirow[t]{2}{*}{ Target population $-40 \%$} & Total cost & $234,530,043$ & $255,748,944$ & $-21,218,900$ \\
\hline & & Cost per patient & 1808.9 & 1972.6 & -163.7 \\
\hline \multirow[t]{12}{*}{ Spain } & \multirow{2}{*}{$\begin{array}{l}\text { Probability of relapses managed } \\
\text { with a hospitalization }-20 \%\end{array}$} & Total cost & $276,800,251$ & $323,099,853$ & $-46,299,602$ \\
\hline & & Cost per patient & 1370.3 & 1599.5 & -229.2 \\
\hline & \multirow[t]{2}{*}{ Cost of relapses $+20 \%$} & Total cost & $315,295,709$ & $405,073,689$ & $-89,777,980$ \\
\hline & & Cost per patient & 1560.9 & 2005.3 & -444.4 \\
\hline & \multirow[t]{2}{*}{ Cost of relapses $-20 \%$} & Total cost & $267,469,550$ & $309,871,518$ & $-42,401,968$ \\
\hline & & Cost per patient & 1324.1 & 1534.0 & -209.9 \\
\hline & \multirow[t]{2}{*}{ Cost of lurasidone $+10 \%$} & Total cost & $308,564,353$ & $357,472,603$ & $-48,908,251$ \\
\hline & & Cost per patient & 1527.5 & 1769.7 & -242.1 \\
\hline & \multirow[t]{2}{*}{ Cost of lurasidone $-10 \%$} & Total cost & $274,635,509$ & $357,472,603$ & $-83,271,697$ \\
\hline & & Cost per patient & 1357.4 & 1769.7 & -412.2 \\
\hline & \multirow[t]{2}{*}{ Target population $-40 \%$} & Total cost & $174,829,578$ & $214,483,562$ & $-39,653,984$ \\
\hline & & Cost per patient & 1442.5 & 1769.7 & -327.2 \\
\hline
\end{tabular}

to a reduction in costs of US\$ -3276 per patient compared with quetiapine XR.

There are limitations to our analysis. The quantification of direct medical costs related to relapses is based on the most recent analyses conducted in the two contexts investigated, published before 2010 . The clinical practice in terms of relapse management may have changed over the years. The uncertainty over this aspect has been managed in the sensitivity analysis, showing a limited impact on the differential cost between the two scenarios. Furthermore, the target population considered in the analysis does not reflect the real number of patients who will be treated with lurasidone or quetiapine XR in each national context, but the results approximate the potential impact of the use of lurasidone in the case where all eligible patients are treated with lurasidone, compared with quetiapine XR.

The main strength of the analysis is related to the use of clinical inputs derived from a direct comparison of lurasidone and quetiapine XR. In detail, the probability of relapse at 12 months (33.6\% for quetiapine XR and 23.7\% for lurasidone) and the probability of hospitalization at 12 months $(23.1 \%$ for quetiapine XR and $9.8 \%$ for lurasidone) were derived from the analysis published by Loebel and colleagues [11], while the probability of relapse managed in an outpatient setting at 12 months was calculated as the difference of the aforementioned probabilities $(10.5 \%$ for quetiapine XR and $11.9 \%$ for lurasidone).

The relevance of our article stems from the fact that there is a lack of studies relating to the economic impact of different treatments for patients affected with schizophrenia and their relapses in countries with a National Health Service such as Italy or Spain, taking into account the management of the relapse with or without hospitalization. We provide a piece of research to fill this gap in the literature. Our finding shows that the use of lurasidone $(74 \mathrm{mg}$ ) for the treatment of patients affected with schizophrenia, compared with quetiapine XR $(300 \mathrm{mg})$, would be a cost-saving strategy in the two contexts investigated assuming the National Health Service point of view. Our literature review has only found a few publications with useful information on that aim, and these are considerably old. As a consequence, further research is needed on this topic. 
Fig. 4 Tornado diagram related to the Italian context

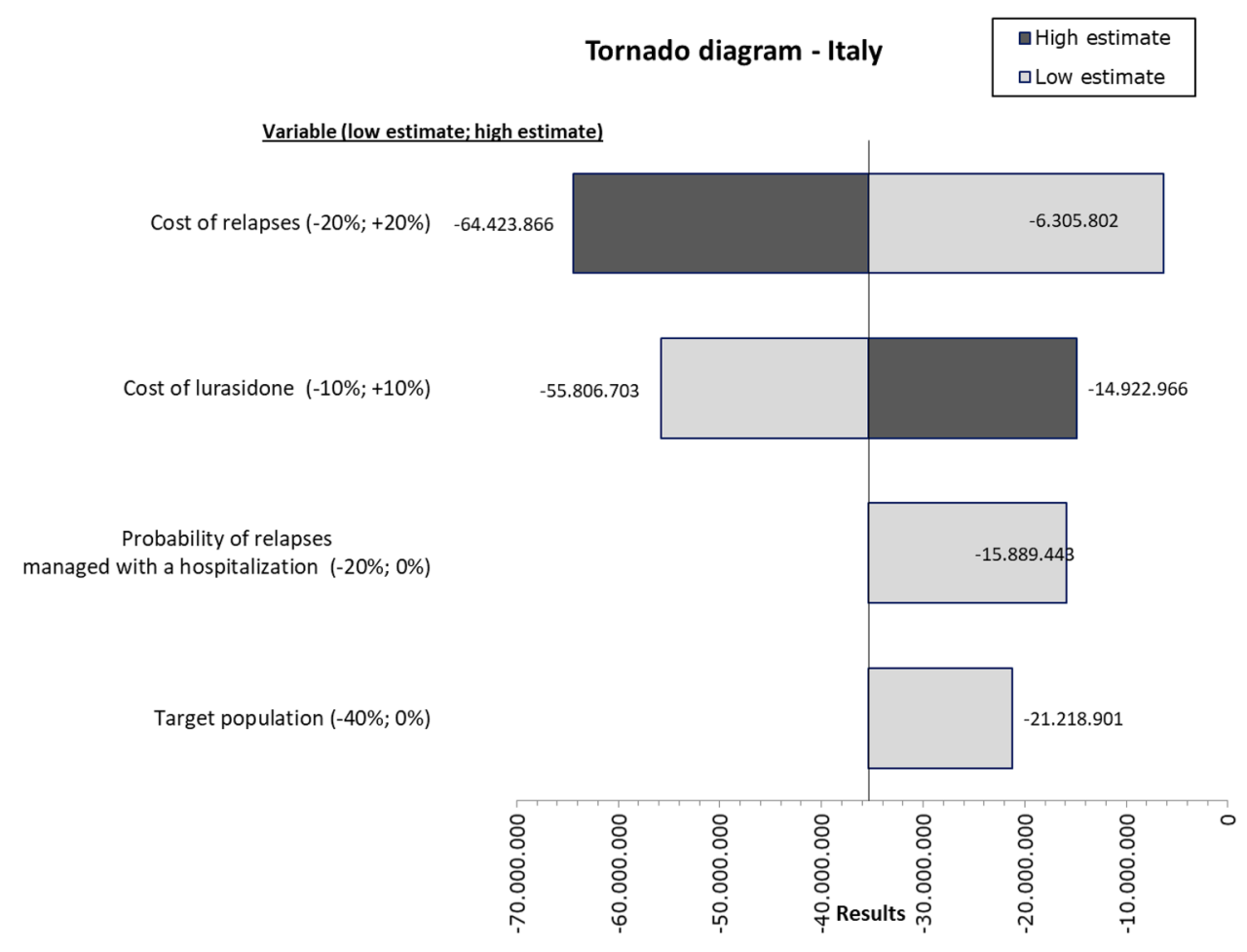

Tornado diagram - Spain

口High estimate

口Low estimate

Fig. 5 Tornado diagram related to the Spanish context

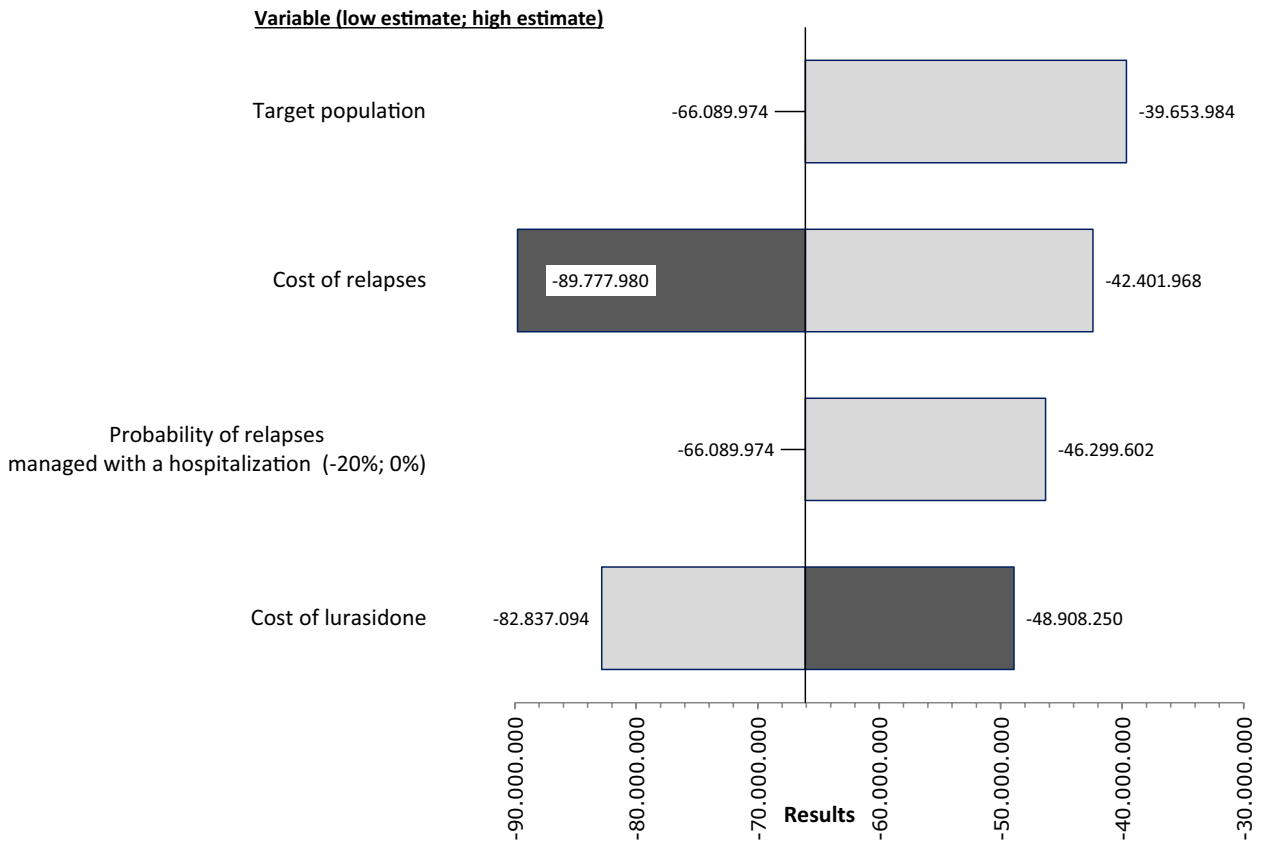

\section{Conclusions}

This article presents an analysis in which we show a reduction in terms of direct medical costs related to the use of lurasidone (74 mg) compared with quetiapine XR (300 mg) in a 1-year time horizon, assuming the point of view of the National Health Service in Italy and Spain. We use the fact that the main cost driver for patients affected with schizophrenia is the probability of experiencing relapses, and our 
analysis is based on the study of the cost of relapses for patients following both treatments. The use of lurasidone compared with quetiapine XR leads to a decrease in relapses with hospitalization in adult patients affected with schizophrenia and the related costs, and to an increase in costs related to relapses without hospitalization and therapy costs. The results show an annual reduction in direct medical costs per patient associated with the use of lurasidone of $-163.7 €$ (with a reduction of $-35.4 €$ million considering the eligible population; $-9.0 \%$ ) in Italy and $-327.2 €$ (with a reduction of $-66.1 €$ million considering the eligible population; $-22.7 \%$ ) in Spain.

Author contributions UR, SS, and DC designed the study; all authors contributed to the model design; UR and SS implemented the model; all authors contributed to the data analysis and interpretation; UR and SS drafted the manuscript and all authors critically revised it for important intellectual content and approved the final submitted version of the manuscript.

\section{Compliance with Ethical Standards}

Funding This analysis was funded by an unconditional grant from Angelini SpA.

Conflict of interest Umberto Restelli, Davide Croce, and Sofia Silvola declare grants received by one of their institutions to conduct the analysis. Manuel García-Goñi participated through his university in a research project financed by Angelini whose result is the manuscript. Benedicto Crespo-Facorro declares advisory board honoraria from Lundbeck, Otsuka, Cassen Recordati, Teva Neuroscience, and Angelini; and speaker's honoraria from Lundbeck, Otsuka, and Janssen Cilag. Michal Lew-Starowicz declares advisory board honoraria from Novo Nordisk; travel/meeting expenses and manuscript preparation and reviewing supported by CREMS Srl, and receiving honoraria for lectures from Abbvie, Angelini, Autrovitas Apotex, CROS-CRO, MCO International Srl, Mylan Healthcare, Lundbeck, Sanofi, and Servier. Pawel Mierzejewski, Jacqueline Mayoral-van Son, and Paola Rocca have no conflicts of interest that are directly relevant to the content of this article.

Data Availability All data used in the analysis are already available in the literature and data sources are reported in the article.

Open Access This article is licensed under a Creative Commons Attribution-NonCommercial 4.0 International License, which permits any non-commercial use, sharing, adaptation, distribution and reproduction in any medium or format, as long as you give appropriate credit to the original author(s) and the source, provide a link to the Creative Commons licence, and indicate if changes were made. The images or other third party material in this article are included in the article's Creative Commons licence, unless indicated otherwise in a credit line to the material. If material is not included in the article's Creative Commons licence and your intended use is not permitted by statutory regulation or exceeds the permitted use, you will need to obtain permission directly from the copyright holder. To view a copy of this licence, visit http://creativecommons.org/licenses/by-nc/4.0/.

\section{References}

1. Charlson FJ, Ferrari AJ, Santomauro DF, Diminic S, Stockings E, Scott JG, et al. Global epidemiology and burden of schizophrenia: findings from the Global Burden of Disease Study 2016. Schizophr Bull. 2018;44(6):1195-203.

2. Kovács G, Almási T, Millier A, Toumi M, Horváth M, Kóczián $\mathrm{K}$, et al. Direct healthcare cost of schizophrenia: European overview. Eur Psychiatry. 2018;48:79-92. https://doi.org/10.1016/j. eurpsy.2017.10.008.

3. Leucht S, Corves C, Arbter D, Engel RR, Li C, Davis JM. Secondgeneration versus first-generation antipsychotic drugs for schizophrenia: a meta-analysis. Lancet. 2009;373(9657):31-41.

4. Gómez-Revuelta M, Pelayo-Terán JM, Juncal-Ruiz M, VázquezBourgon J, Suárez-Pinilla P, Romero-Jiménez R, et al. Antipsychotic treatment effectiveness in first episode of psychosis: PAFIP 3 -year follow-up randomized clinical trials comparing haloperidol, olanzapine, risperidone, aripiprazole, quetiapine and ziprasidone. Int J Neuropsychopharmacol. 2020;23(4):217-29.

5. Rajagopalan K, O’Day K, Meyer K, Pikalov A, Loebel A. Annual cost of relapses and relapse-related hospitalizations in adults with schizophrenia: results from a 12-month, double-blind, comparative study of lurasidone vs quetiapine extended-release. J Med Econ. 2013;16(8):987-96.

6. Mayoral-van Son J, Juncal-Ruiz M, Ortiz-García de la Foz V, Cantarero-Prieto D, Blázquez-Fernández C, Paz-Zulueta M, et al. Understanding the direct and indirect costs of a first episode of psychosis program: Insights from PAFIP of Cantabria, Spain, during the first year of intervention. Early Interv Psychiatry. 2019;13(5):1182-90.

7. Olivares JM, Rodriguez-Martinez A, Burón JA, Alonso-Escolano D, Rodriguez-Morales A, e-STAR Study Group. Cost-effectiveness analysis of switching antipsychotic medication to long-acting injectable risperidone in patients with schizophrenia: a 12- and 24-month follow-up from the e-STAR database in Spain. Appl Health Econ Health Policy. 2008;6(1):41-53.

8. Gutiérrez-Recacha P, Chisholm D, Haro JM, Salvador-Carulla L, Ayuso-Mateos JL. Cost-effectiveness of different clinical interventions for reducing the burden of schizophrenia in Spain. Acta Psychiatr Scand Suppl. 2006;432:29-38.

9. Caseiro O, Pérez-Iglesias R, Mata I, Martínez-Garcia O, PelayoTerán JM, Tabares-Seisdedos R, et al. Predicting relapse after a first episode of non-affective psychosis: a three-year follow-up study. J Psychiatr Res. 2012;46(8):1099-105.

10. Hasan A, Falkai P, Wobrock T, Lieberman J, Glenthoj B, Gattaz WF, World Federation of Societies of Biological Psychiatry (WFSBP) Task Force on Treatment Guidelines for Schizophrenia, et al. World Federation of Societies of Biological Psychiatry (WFSBP) guidelines for biological treatment of schizophrenia, part 1: update 2012 on the acute treatment of schizophrenia and the management of treatment resistance. World J Biol Psychiatry. 2012;13(5):318-78.

11. Loebel A, Cucchiaro J, Xu J, Sarma K, Pikalov A, Kane JM. Effectiveness of lurasidone vs. quetiapine XR for relapse prevention in schizophrenia: a 12-month double-blind noninferiority study. Schizophr Res. 2013;147(1):95-102.

12. European Medicines Agency. Latuda: lurasidone. 2014. https:// www.ema.europa.eu/en/medicines/human/EPAR/latuda. Accessed 23 June 2020.

13. Greenberg WM, Citrome L. Pharmacokinetics and pharmacodynamics of lurasidone hydrochloride, a second-generation antipsychotic: a systematic review of the published literature. Clin Pharmacokinet. 2017;56(5):493-503.

14. European Medicines Agency. Questions and answers on Seroquel XR and associated names (50, 150, 200, 300 and $400 \mathrm{mg}$ 
prolonged-release tablets containing quetiapine). 2010. https:// www.ema.europa.eu/en/documents/referral/questions-answersseroquel-xr-associated-names-50-150-200-300-400-mg-prolo nged-release-tablets_en.pdf. Accessed 23 June 2020.

15. DeVane CL, Nemeroff CB. Clinical pharmacokinetics of quetiapine: an atypical antipsychotic. Clin Pharmacokinet. 2001;40:509-22.

16. Italian Ministry of Health. Rapporto salute mentale. Analisi dei dati del Sistema Informativo per la Salute Mentale (SISM) Anno 2017. December 2018. http://www.salute.gov.it/imgs/C_17_pubbl icazioni_2841_allegato.pdf. Accessed 25 June 2020.

17. Eurostat. Population on 1 January. 2019. https://ec.europa.eu/ eurostat/databrowser/view/tps00001/default/table?lang=en. Accessed 23 June 2020.

18. Rubio-Terrés C, Montejo González AL, Puchol Incertis M, Álvarez SC. Análisis farmacoeconómico del cambio de tratamiento antipsicótico, por ineficacia o efectos adversos, a quetiapina de liberación prolongada. Pharmacoecon Span Res Artic. 2009;6:105-14. https://doi.org/10.1007/BF03320858.

19. Lacro JP, Dunn LB, Dolder CR, Leckband SG, Jeste DV. Prevalence of and risk factors for medication nonadherence in patients with schizophrenia: a comprehensive review of recent literature. J Clin Psychiatry. 2002;63(10):892-909.
20. Moher D, Liberati A, Tetzlaff J, Altman DG, PRISMA Group. Preferred reporting items for systematic reviews and meta-analyses: the PRISMA statement. PLoS Med. 2009;6(7):e1000097.

21. Dilla T, Möller J, O’Donohoe P, Álvarez M, Sacristán JA, Happich M, Tockhorn A. Long-acting olanzapine versus long-acting risperidone for schizophrenia in Spain: a cost-effectiveness comparison. BMC Psychiatry. 2014;2(14):298.

22. Peirò S, Gómez G, Navarro M, Guadarrama I, Rejas J, Psychosp Group. Length of stay and antipsychotic treatment costs of patients with acute psychosis admitted to hospital in Spain. Soc Psychiatry Psychiatr Epidemiol. 2004;39(7):507-13.

23. Berto P, Negrini C, Ferrannini L. Analisi costo-efficacia di paliperidone ER nel trattamento delle ricadute della schizofrenia, nella prospettiva del Sistema Sanitario Nazionale italiano. Farmacoeconomia e Percorsi Clinici. 2008;9(2):95-108.

24. International Monetary Fund. Inflation average consumer prices: Italy and Spain. 2019. https://www.imf.org/external/datamapper/ PCPIPCH@WEO/OEMDC/ITA/ESP. Accessed 23 June 2020.

25. Leucht S, Samara M, Heres S, Patel MX, Woods SW, Davis JM. Dose equivalents for second-generation antipsychotics: the minimum effective dose method. Schizophr Bull. 2014;40(2):314-26.

\section{Affiliations}

\section{Umberto Restelli ${ }^{1,2}$ - Manuel García-Goñi ${ }^{3} \cdot$ Michal Lew-Starowicz ${ }^{4,5} \cdot$ Pawel Mierzejewski $^{6} \cdot$ Sofia Silvola ${ }^{1}$. Jacqueline Mayoral-van Son ${ }^{7}$. Davide Croce ${ }^{1} \cdot$ Paola Rocca $^{8} \cdot$ Benedicto Crespo-Facorro $^{7}$}

\author{
Manuel García-Goñi \\ mggoni@ucm.es \\ Michal Lew-Starowicz \\ michallew-starowicz@wp.pl \\ Pawel Mierzejewski \\ pawelmierzej@yahoo.com \\ Sofia Silvola \\ silvola.sofia@gmail.com \\ Jacqueline Mayoral-van Son \\ jacquelinemvs@yahoo.es \\ Davide Croce \\ d.croce@tiscali.it \\ Paola Rocca \\ paola.rocca@unito.it \\ Benedicto Crespo-Facorro \\ benedicto.crespo@unican.es
}

1 Center for Health Economics, Social and Health Care Management, University Carlo Cattaneo-LIUC, Corso Matteotti, 22, 21053 Castellanza, VA, Italy

2 School of Public Health, Faculty of Health Sciences, University of the Witwatersrand, Johannesburg, South Africa

3 Department of Applied and Structural Economics and History, Faculty of Economics and Business, Universidad Complutense de Madrid, Madrid, Spain

4 Department of Psychiatry, Centre of Postgraduate Medical Education, Warsaw, Poland

5 Institute of Psychiatry and Neurology, Warsaw, Poland

6 Department of Pharmacology, Institute of Psychiatry and Neurology, Warsaw, Poland

7 University Hospital Virgen del Rocio, University of Sevilla, CIBERSAM, Seville, Spain

8 Department of Neuroscience, University of Turin, Turin, Italy 